\title{
ERRATUM
}

ERRATUM

Corrigendum to Volume 113, Number 2, p 278-288

(c) Springer-Verlag 2009

\section{Postsurgical diagnostic evaluation of patients with differentiated thyroid carcinoma: comparison of ultrasound, iodine-131 scintigraphy and PET with fluorine-18 fluorodeoxyglucose}

Valutazione diagnostica post-chirurgica di pazienti con carcinoma tiroideo differenziato: confronto tra ecografia, scintigrafia con iodio-131 e PET con fluoro-18 fluorodesossiglucosio

\author{
O. Caleo • S. Maurea • M. Klain • B. Salvatore • G. Storto • M. Mancini • L. Pace • M. Salvatore
}

Dipartimento di Scienze Biomorfologiche e Funzionali (DSBMF), Università degli Studi di Napoli Federico II (UNINA), Istituto di Biostrutture e Bioimmagini (IBB), Consiglio Nazionale delle Ricerche (CNR), Fondazione SDN (IRCCS), Napoli, Italy

In the article unfortunately authors' affiliation was incomplete

The original version of the article can be found at:

http://dx.doi.org/10.1007/s11547-008-0243-z 